\title{
Enhancing Respiratory Infection Surveillance on the US/Mexico Border- Arizona BIDS Program Sentinel Surveillance Data
}

\author{
Orion McCotter*1, 2, Zimy Wansuala ${ }^{1}$, Catherine Golenko ${ }^{1}$, Natalie Whitfield ${ }^{2}$, Mariana \\ Casal $^{1}$, Jonathan Schouest ${ }^{1}$, Robert Guerrero ${ }^{1}$, Nubia Hernandez ${ }^{3}$, Sergio O. Alba ${ }^{3}$ and \\ Francisco N. Galvez ${ }^{3}$
}

${ }^{1}$ Office of Border Health, Arizona Department of Health Services, Tucson, AZ, USA; ${ }^{2}$ University of Arizona Medical Center, Tucson, AZ, USA; ${ }^{3}$ Secretaria de Salud de Sonora, Sonora, Mexico

\section{Objective}

To present the epidemiology, clinical aspects, and laboratory results of AZ SARI case patients and to describe respiratory viruses in the $\mathrm{AZ}$ border region.

\section{Introduction}

This project was established through the Border Infectious Disease Surveillance (BIDS) program in Arizona (AZ) to monitor infecting respiratory pathogens among hospitalized patients with Severe Acute Respiratory Infections (SARI) in the AZ border region from September 2010 to the present.

\section{Methods}

This SARI surveillance is conducted by BIDS at three acute care hospitals in the AZ border region. Each site enrolls and collects specimens and clinical data on patients meeting the SARI case definition. A SARI case is defined as a patient requiring hospital admission with fever (self-reported or measured $\geq 37.8^{\circ} \mathrm{C}$ ) and cough or sore throat. Viral, bacterial, and fungal tests are performed for each enrolled SARI case using extensive laboratory techniques; some case patients may have additional testing such as rapid influenza diagnostic testing (RIDT) and chest x-ray imaging results if they were ordered separately by the physician.

\section{Results}

A total of 175 patients meeting SARI case definition have been enrolled in BIDS SARI surveillance from project onset in September 2010 through August 2013. Patients who were 65 years and older comprised the largest age group, Hispanic patients comprised the largest race/ethnicity category, and the majority of cases were male. The most commonly identified pathogens among the positive viral SARI specimens were: RSV, influenza, common cold viruses (rhinovirus, coxsackievirus, and echovirus), parainfluenza, and human metapneumovirus. Among a subset of SARI case patients with additional RIDT data available ( $\mathrm{n}=64)$, ten patients initially negative by RIDT were later confirmed influenza positive by reverse-transcriptase polymerase chain reaction (RT-PCR) testing.

\section{Conclusions}

Enhanced laboratory data from SARI case patients can be used to examine respiratory virus distribution, identify novel strains, and aid in the development of public health strategies for infectious disease prevention.

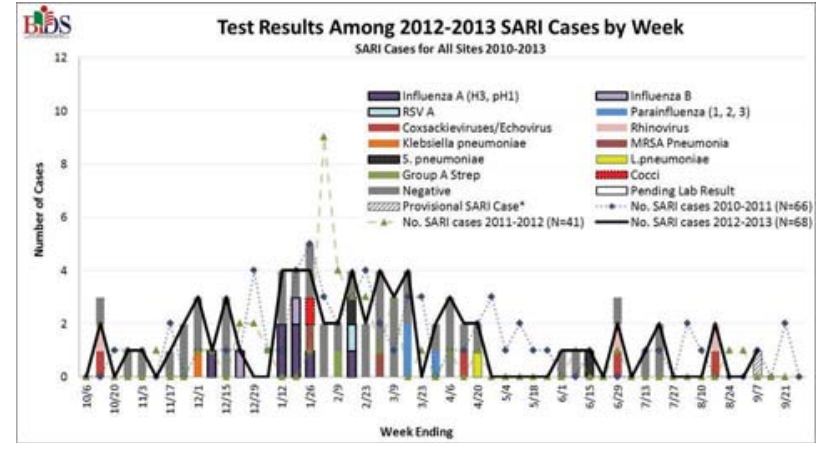

Keywords

respiratory; surveillance; border; influenza; syndrome

*Orion McCotter

E-mail: Orion.McCotter@azdhs.gov 